\title{
Identification and phylogenetic analyses of VASt, an uncharacterized protein domain associated with lipid-binding domains in Eukaryotes
}

\author{
Mehdi Khafif ${ }^{1,2}$, Ludovic Cottret $^{1,2}$, Claudine Balagué ${ }^{1,2}$ and Sylvain Raffaele ${ }^{1,2^{*}}$
}

\begin{abstract}
Background: Several regulators of programmed cell death (PCD) in plants encode proteins with putative lipidbinding domains. Among them, VAD1 is a regulator of PCD propagation harboring a GRAM putative lipid-binding domain. However the function of VAD1 at the subcellular level is unknown and the domain architecture of VAD1 has not been analyzed in details.

Results: We analyzed sequence conservation across the plant kingdom in the VAD1 protein and identified an uncharacterized VASt (VAD1 Analog of StAR-related lipid transfer) domain. Using profile hidden Markov models (profile HMMs) and phylogenetic analysis we found that this domain is conserved among eukaryotes and generally associates with various lipid-binding domains. Proteins containing both a GRAM and a VASt domain include notably the yeast Ysp2 cell death regulator and numerous uncharacterized proteins. Using structure-based phylogeny, we found that the VASt domain is structurally related to Bet v1-like domains.

Conclusion: We identified a novel protein domain ubiquitous in Eukaryotic genomes and belonging to the Bet v1-like superfamily. Our findings open perspectives for the functional analysis of VASt-containing proteins and the characterization of novel mechanisms regulating PCD.
\end{abstract}

Keywords: VASt, VAD1, Protein domain, Programmed cell death, GRAM domain, Bet v1-like

\section{Background}

Protein domain predictions are a starting point for a range of functional analyses and can either newly predict or further refine functional predictions [1]. Indeed, domains form structural, evolutionary and functional units of proteins [2]. The combination and order of domains in a protein is frequently considered as a fundamental level of protein functional complexity. The majority of proteins is composed of multidomain proteins and the domain composition of multidomain proteins is critical for their specialized functions [3]. Furthermore, domain combinations are not random, which may indicate functional cooperation [4].

\footnotetext{
* Correspondence: Sylvain.raffaele@toulouse.inra.fr

${ }^{1}$ INRA, Laboratoire des Interactions Plantes-Microorganismes (LIPM), UMR441, 24 Chemin de Borde Rouge - Auzeville, CS52627, F31326 Castanet Tolosan Cedex, France

${ }^{2}$ CNRS, Laboratoire des Interactions Plantes-Microorganismes (LIPM), UMR2594, 24 Chemin de Borde Rouge - Auzeville, CS52627, F31326 Castanet Tolosan Cedex, France
}

Plant "lesion mimic mutants" (LMMs) show spontaneous necrotic lesion resembling the so-called Hypersensitive Response (HR), a form of programmed cell death associated with plant defense [5,6]. The Arabidopsis thaliana vad1 (vascular associated death1) mutant is a LMM altered in a negative regulator of $\mathrm{PCD}$ and defense responses harboring a GRAM domain predicted to bind lipids $[7,8]$. Contrary to most LMM genes characterized to date, VAD1 is expected to control the cell-to-cell propagation of PCD instead of its initiation [5]. GRAM is a $\sim 70$ amino-acids domain predicted to mediate intracellular protein binding or lipid binding during membrane-associated processes [9]. This domain is related to the PH domains and is found in animal glucosyltransferases, Rab-like GTPase activators, myotubularins and other membrane-associated proteins. The GRAM domain of human myotubularins is able to bind phospholipids and is involved in membrane signaling [10-12], but its exact function often remains 
enigmatic [13].The presence of a GRAM domain in VAD1 protein suggests that lipid binding could be required for VAD1 function, but the role of VAD1 at the subcellular level is currently unknown.

Remarkably, a significant proportion of plant LMMs show mutations in genes associated with lipid biosynthesis and homeostasis [14-17]. This notably concerns sphingolipid metabolism: the acd5 and acd11 mutants carry mutations in a ceramide kinase and a putative sphingosine transfer protein, respectively $[15,16]$. Conversely, $E R H 1$ is a positive regulator of the $\mathrm{HR}$ encoding a functional inositolphosphoryl-ceramide (IPC) synthase which converts ceramide to IPC [18]. Furthermore, EDR2 was isolated as a negative regulator of $\mathrm{PCD}$ and defense responses encoding a multi-domain protein featuring a DUF1336 domain, a PH domain and a START domain $[17,19]$. Like CERT PH domain, EDR2 PH domain preferentially binds to PI4P [19]. Nevertheless, the mechanisms by which lipid-binding domain containing proteins regulate PCD in plants are largely unknown.

Here, we analyzed sequence conservation in the VAD1 family and identified an uncharacterized conserved domain we designated as VASt (VAD1 Analog of StAR-related lipid transfer). Using sequence- and structure-based phylogenetic analyses we demonstrate that this domain is present in all major eukaryotic lineages but no molecular function has been assigned to it. VASt is related to Bet v1-like, a superfamily including lipid- and hormone-binding domains. The VASt domain will be referred to with accession number PF16016 in release 28.0 of the Pfam database [20]. These findings open new perspectives for the functional analysis of VASt-domain containing proteins such as $A$. thaliana VAD1, yeast YSP2 and human GRAM1A, B and $\mathrm{C}$.

\section{Results}

Proteins in the VAD1 family contain an uncharacterized conserved domain

To get insights into VAD1 putative biochemical function, we analyzed protein sequence conservation among VAD1 homologs. First, to identify VAD1 homologs, we used the full length sequence of VAD1 protein in stringent phmmer searches against the Uniprot database. We identified 13 AtVAD1 homologs with e-value below $1 \mathrm{e}^{-100}$ across twelve angiosperm plant species, including monocots (Brachypodium distachyon, Musa acuminata, Oryza brachyantha, Oryza sativa, Setaria italica, Sorghum bicolor) and eudicots (Arabidopsis lyrata, Glycine max, Ricinus communis, Solanum lycopersicum, Vitis vinifera). In this stringent approach, all species showed a single VAD1 copy except G. max that had three copies. The retrieved homologs showed at least $55 \%$ identity and all contained a clearly identified GRAM domain. To identify conserved regions in these 14 sequences, we aligned them using the Multiple Sequence Alignment (MSA) tool MAFFT [21] and we plotted the consensus conservation score along the alignment using a ten Amino-Acids (AA) sliding window (Figure 1A). Two major conserved regions were clearly apparent. The first one spanning positions 80 to 200 in the alignment, and the second spanning positions 300 to 480 . To characterize and precisely delimit VAD1 conserved regions we mapped VAD1 gene and protein annotations onto the conservation plot (Figure 1B).

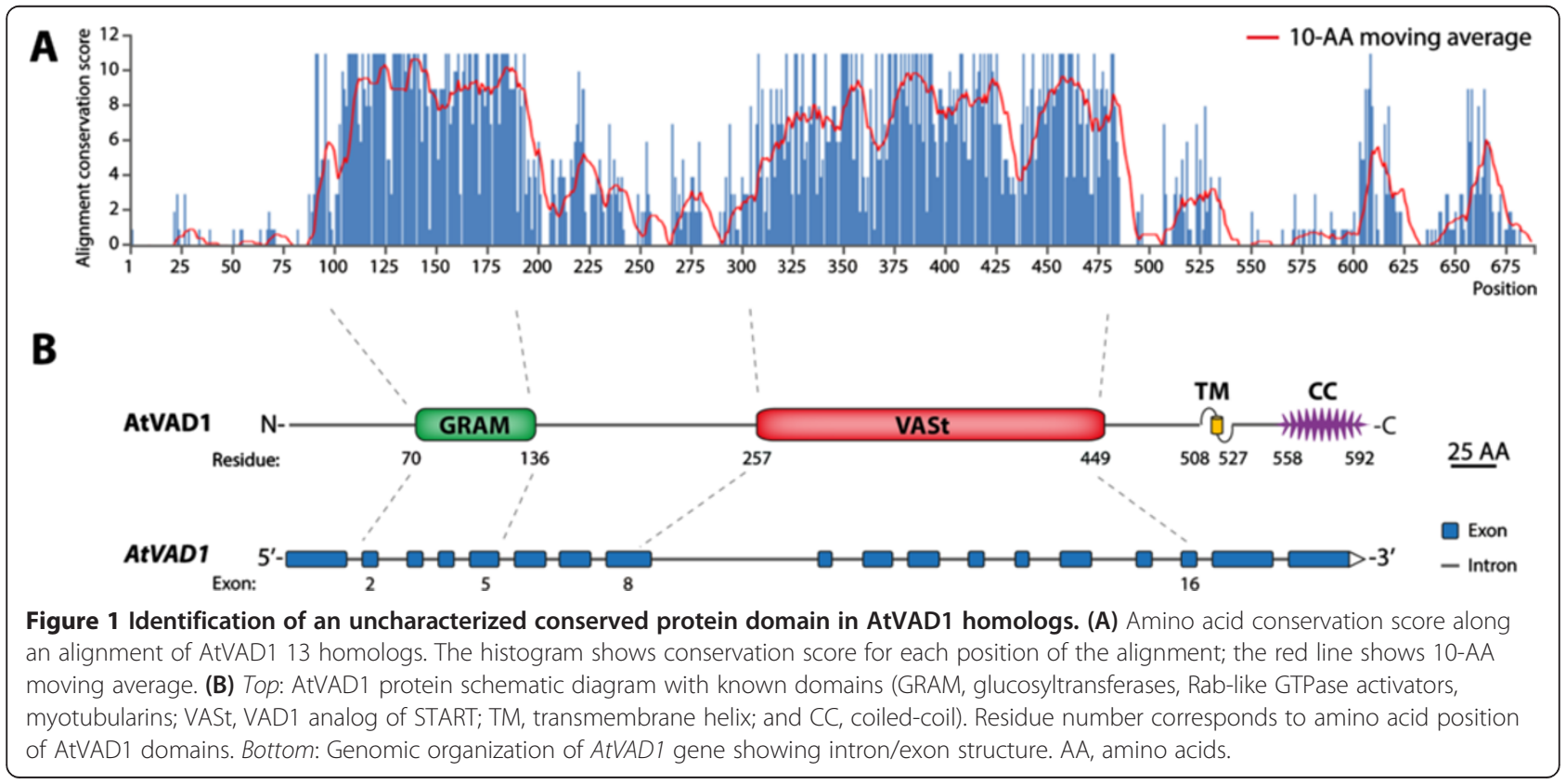


The N-terminal conserved region (position 80-200) overlapped largely with the predicted GRAM domain (position 116-182). The second conserved region (position 300-480) corresponded to an uncharacterized domain of approximately 190 AA. This domain is encoded by a region spanning from AtVAD1 exon 8 to exon 16 . We therefore set the limits of this uncharacterized domain at positions 257 and 449, for a total length of 193 AA (Figure 1B). Based on further characterization described hereafter, we designated this domain as the VASt (VAD1 Analog of START) domain. A close up view on the MSA of the VASt domain revealed a high degree of conservation among plant homologs, with an average $70.5 \%$ identity over the VASt domain (Additional file 1: Figure S1, Additional file 2). Since no annotation could be mapped onto the VASt domain, it represents a yet uncharacterized protein domain highly conserved in plants.

\section{The VASt domain is conserved among Eukaryotes}

Since none of AtVAD1 homologs had characterized biochemical functions, we extended our search of related protein domains using Hidden Markov Models (HMM) to get insights into the VASt domain putative function and evolution. For this, we built a HMM using the 13 plant homologs of AtVAD1 VASt domain. To highlight important sequence motifs in this HMM, we examined the corresponding sequence logo (Figure 2A). Amino acids W61, R71, P116, F121 and I160 were strongly conserved in the model, suggesting an important contribution of these residues to the protein function. To identify protein domains related to the VASt domain, we searched the Uniprot database with the VASt HMM model. After three iterations of jackhammer search, we retrieved 452 hits distributed exclusively in eukaryotic proteins, including yeasts and other Fungi, Oomycetes, Mammals, and Plants (Additional file 3). Most of the identified proteins contained one copy of the VASt domain ( 85.8\%), with a few containing two or more VASt domains (Figure 2B). As little as $\sim 21.9 \%$ of the protein hits contained VASt as the only domain identified. The VASt domain is frequently associated with lipid binding domains such as GRAM (in $70.3 \%$ of the hits), C2 and Pex24p domains, suggesting a functional link between VASt and lipid-binding domains. Among proteins related to VAD1 retrieved by our HMM search was YSP2, a major cell death regulator in Yeast [22]. However, there was no protein for which a biochemical function has been described.

\section{Multiple domain combinations contributed to the diversification of VASt-containing proteins}

To document the evolution of the VASt domain, we examined the phylogenetic relations of VASt domains in 17 fully sequenced species representing all major Eukaryotic lineages (see Methods), corresponding to a total of 85 protein sequences (after redundancy and incomplete sequence filtering). (Figure 3, Additional file 4, Additional file 5, Additional file 6). Sequences clustered into nine groups defined by their taxonomic range and domain organization. To highlight the phylogenetic relationship between copies of the VASt domain present in a single protein (clades 1, 2, 4 and 5), we have connected together VASt copies present in the same protein (Figure 3A). Group 1 gathered sequences from mammals and other metazoans. In this group, the VASt domain is found either (i) alone, (ii) associated with a GRAM domain or (iii) associated with another VASt domain. The two copies of VASt in GRAM-VASt-VASt proteins clustered in the same clade indicating that the duplication of VASt domain is recent. This group included human GRAMD1A, GRAMD1B and GRAMD1C proteins. Group 2 exclusively consisted in sequences from fungi, with the same domain structures as found in Group 1. Group 2 contained the yeast YSP2 protein. Group 3 and 8 contained $\mathrm{N}$-terminal and $\mathrm{C}$-terminal VASt domains respectively of proteins with a C2-VASt(C2)-GRAM-VASt domain architecture. Group 3 and 8 are restricted to plants, including the moss Physcomitrella patens, and phylogenetically distant, suggesting that duplication of the VASt domain early in land plant evolution allowed the divergence of two VASt copies in these proteins. Group 4 contained proteins from Stramenopiles, Ciliates and Amoebozoa, with either a VASt domain alone or a GRAM-VASt architecture. Group 5 contained exclusively plant sequences with a GRAM-VASt structure, include $A$. thaliana VAD1. Group 6 contained exclusively Stramenopile sequences with either a VASt domain alone or a FCH-GRAM-VASt architecture. Group 7 contained exclusively Ciliate sequences comprising either a VASt domain alone, or GRAM domain with one to three copies of VASt. Finally, Group 9 contained sequences from Amoebozoa, Tracheophyta (vascular plants) and Stramenopiles with diverse domain architectures: (i) VASt alone, (ii) GRAM-VASt, (iii) C2-GRAM-VASt or (iv) VASt-VASt-Pex24p. Groups 3, 5, 8 and 9 show support value of 1.0 suggesting that the function of VASt domains from these groups could have diverged, and that this divergence could be essentially driven by the association with the $\mathrm{C} 2$ and the GRAM domains.

Next, we attempted to reconstruct the evolutionary history of domain combinations in VASt-containing proteins (Figure 3B). Similar to GRAM [13], the VASt domain would originate from the last common eukaryote ancestor (LCEA). Since GRAM and VASt domains are associated in nearly all eukaryotic lineages the GRAM-VASt combination probably dates back from the LCEA. Sequences harboring a VASt domain alone were also found in all lineages except Plants and Fungi, suggesting that a copy of the ancestral VASt domain gene has 


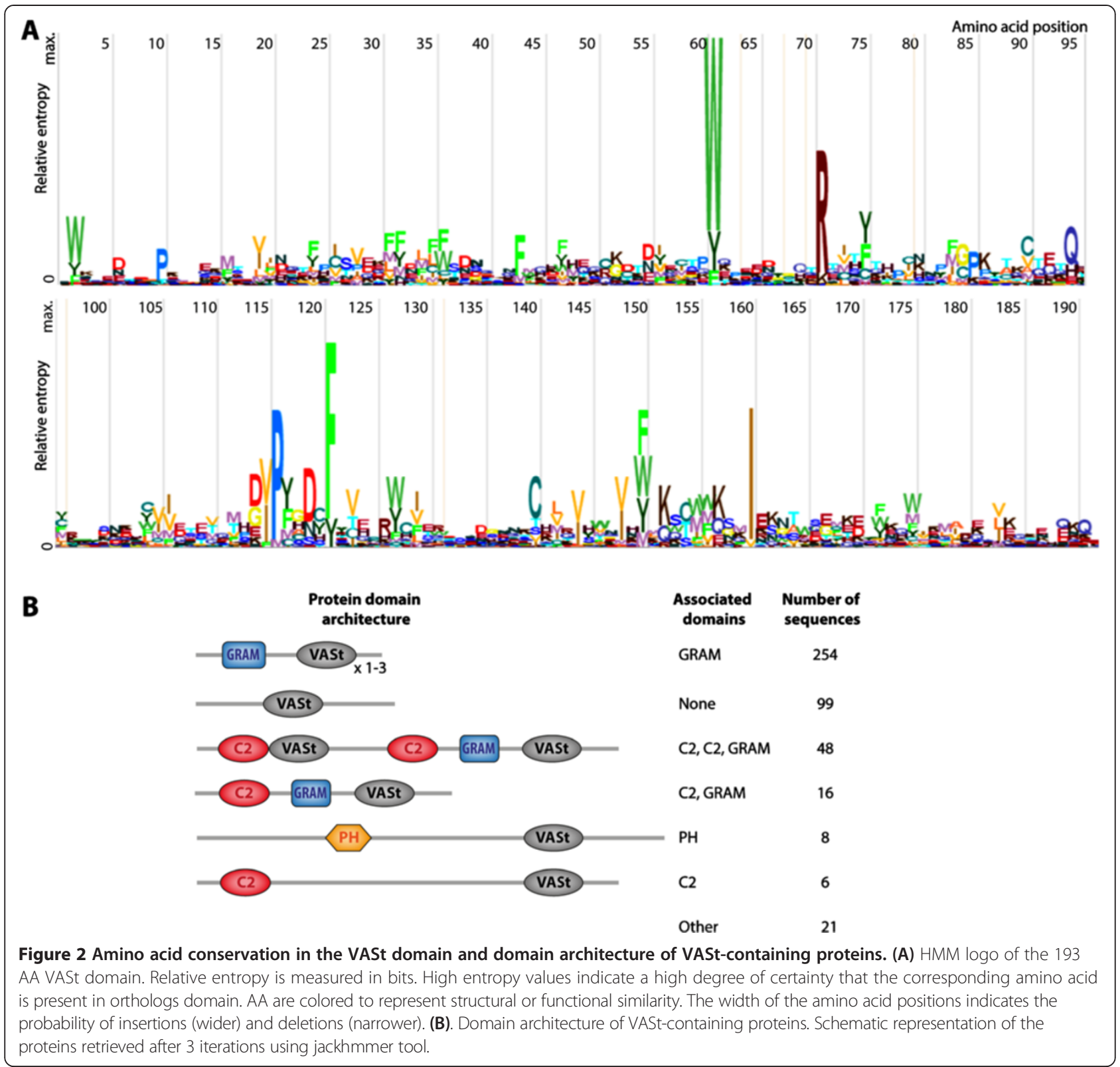

been maintained in most phyla. Alternatively, the GRAM domain could have been lost from a putative GRAM-VASt ancestor in several lineages. Adjacent VASt duplications within a single protein are observed in Ciliates, Stramenopiles, Fungi and Mammals that probably arose recently, judging from high sequence similarity between adjacent copies. Consistent with [13], VASt association with both GRAM and C2 appeared Plantspecific. A parsimonious scenario for the emergence of the complex C2-VASt-C2-GRAM-VASt domain architecture specific to Plants could be the combination of a C2 domain with ancestral VASt alone and GRAM-VASt proteins, followed by the fusion of a C2-VASt and a
C2-GRAM-VASt module early in the evolution of Plants (Figure 3B). The C2-VASt and C2-GRAM-VASt modules could have been maintained in vascular plants but not in mosses. Alternatively, a VASt-GRAM-VASt fusion could have emerged in an ancestral Eukaryotic lineage (it has been maintained in Mammals), combined with two C2 domains in plants, then C2-VASt and C2-GRAM-VASt could have emerged from the split of a C2-VASt-C2-GRAMVASt plant ancestor in vascular plants but not in mosses. The association of VASt with Pex24p or FCH domains seems to be innovations from the Oomycete lineage. In addition to data presented in Figure 3, proteins with a $\mathrm{PH}-\mathrm{VASt}$ architecture were found in some fungal species. 


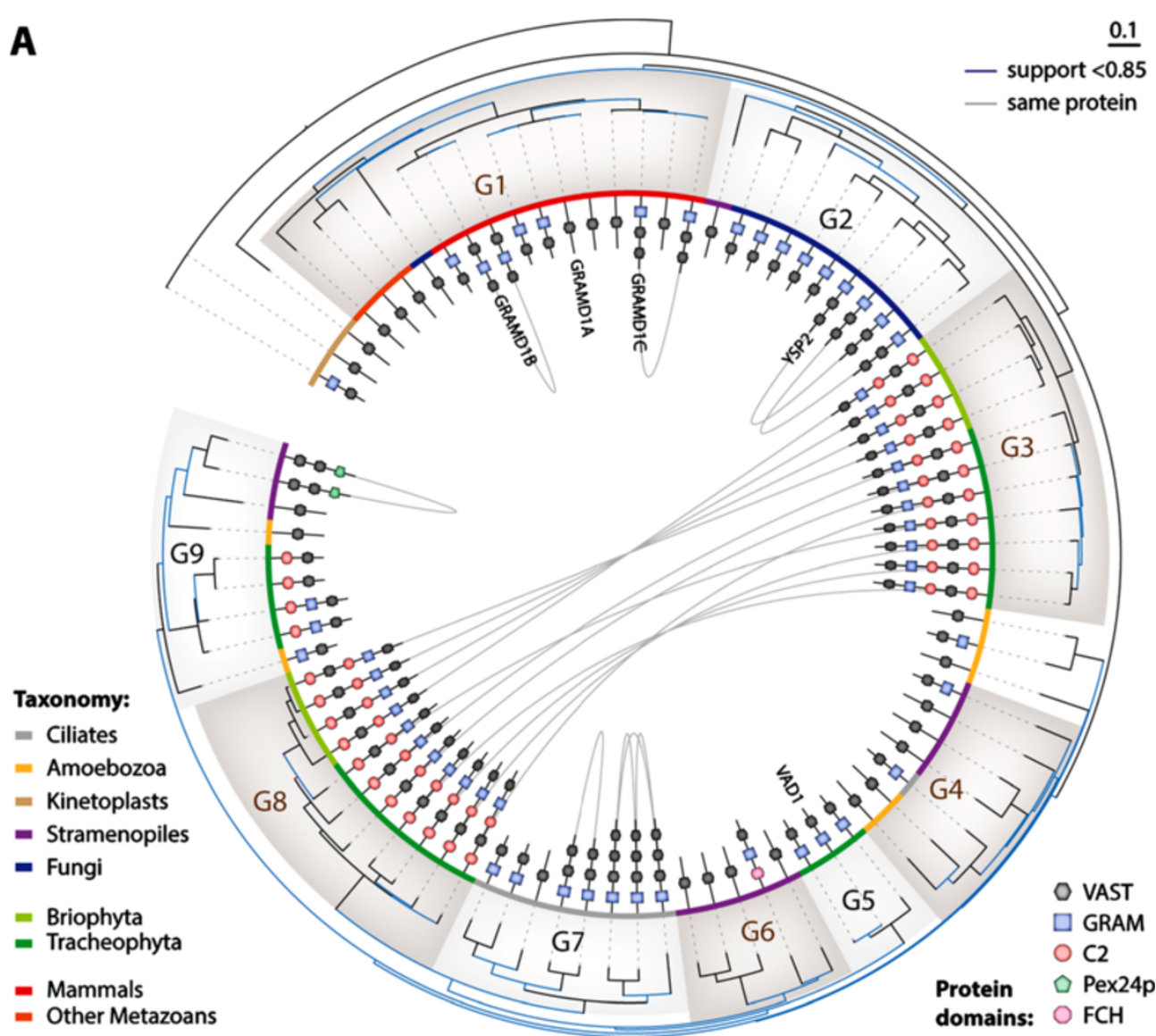

B

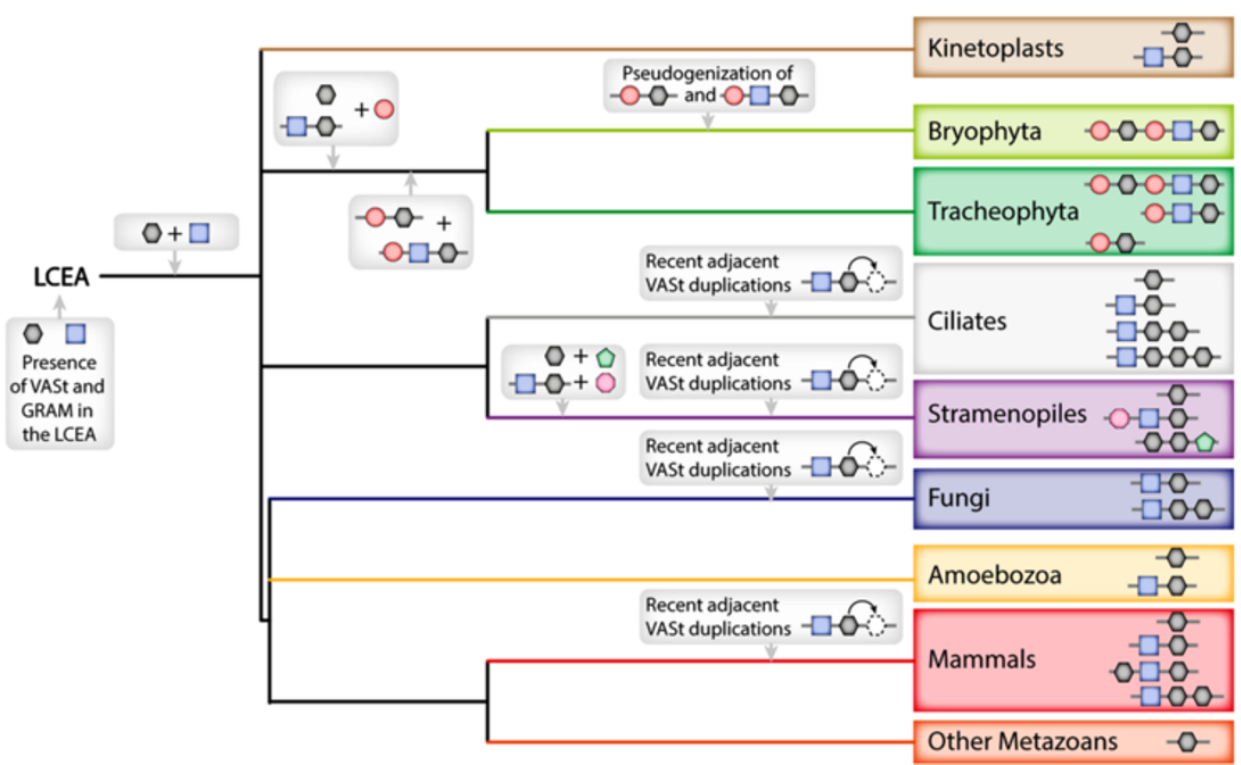

Figure 3 Phylogeny and evolutionary history of the VASt domain. (A) Maximum likelihood phylogenetic tree of 85 VASt protein domains from 17 fully-sequenced species representative of major Eukaryotic lineages. Multiple copies of VASt found in a single protein are linked with central connectors. Protein Domain architecture and taxonomy are shown along branches. Nine phylogenetic groups (G1 to G9) are highlighted. Proteins cited in the main text are labeled along branches. (B) Proposed scenario for the evolution of VASt-containing proteins. Domain symbols are as in $\mathbf{A}$. LCEA, last common Eukaryote ancestor. 
Homology modeling of the VASt domain 3D structure Classical sequence-based phylogeny did not allow identifying protein domains of known function related to VASt. Structure-based phylogenetic network inference may be used to improve the resolution of deep evolutionary relationships and assist in inference of protein function [23]. To analyze relationships between VASt and protein domains of known three-dimensional structure, we conducted a structure-based clustering of AtVAD1 VASt domain and its closest analogs. First, to obtain atomic coordinates of a 3D model for AtVAD1 VASt domain, we submitted its 193 AA sequence to the homology and threading structure prediction server I-TASSER [24]. The best model (Additional file 7) showed a two-layer sandwich alpha beta fold (CATH 3.30, also called "helix grip fold", [25]) containing three alpha helices ( $\alpha 1$ to 3 ), six beta-sheets ( $\beta 1$ to 6$)$ and two loops ( $\Omega 1$ and 2 ) numbered from $N$ to $C$ terminus (Figure 4A). This model had a C-score of -1.41indicating that quality predictions can be estimated with more than 90\% confidence [24], and expected TM-score of 0.54 suggesting a correct topology. Eight of the top 10 threading alignments had normalized Z-score higher than 1, thus accuracy of the model is expected to be high [26]. The predicted VASt model encompasses a large hydrophobic cavity delimited by sheets $\beta 2,3$ and 4, loop $\Omega 1$ and helices $\alpha 2$ and 3 (Figure 4B).

Structure-based phylogeny reveals relationships between the VASt domain and Bet v1-like domains

To investigate structural relationships between AtVAD1 VASt domain and its closest analogs, we conducted a structure-based tree inference analysis including AtVAD1 VASt domain, predicted three-dimensional structure for 15 VASt homologs, and the top structural analogs retrieved by fold recognition searches. The best structural analogs retrieved by I-TASSER and NCBI Vector Alignment Search Tool fold recognition searches were human MLN64 (Metastatic axillary lymph node protein 64) STAR-related lipid transport domain [PDB:1EM2] and Streptomyces ZhuI polyketide aromatase/cyclase [PDB:3TFZ]. Close analogs also included human CERT ceramide trafficking protein [PDB:2E3M] and Arabidopsis thaliana PYL2 ABA receptor [PDB:3KDI]. Gene Ontology terms associated with AtVAD1 VASt domain based on the 3D model included hormone binding [GO:0042562], isoprenoid binding [GO:0019840] and monocarboxylic acid binding [GO:0033293]. To build a structure-based phylogenetic tree, we modeled the three-dimensional structure of 15 VASt domains using AtVAD1 VASt as a template, and searched for AtVAD1 VASt closest structural analogs in the medium redundancy subset of the Molecular Modeling database (Additional file 8). We next performed a multiple structure alignment, calculated normalized pairwise RMSD distances for aligned $\mathrm{C} \alpha$ atoms (Additional file 9), and used this distance matrix to produce a neighbor-joining tree (Additional file 10). Structures clustered into five major groups (Figure 5). Group I contained plant Bet v1 phytohormone-binding proteins and pathogenesis-related (PR) 10-like proteins. Group II contained Arabidopsis thaliana PYL2 abscisic acid (ABA) receptor, belonging to the Pyrabactin resistance 1 (PYR1)/PYR1-like (PYL)/Regulatory components of ABA receptors (RCAR) family and uncharacterized bacterial proteins. Group III contained mammalian START proteins binding sterols and sphingolipids, and Streptomyces aromatase-cyclases. Group IV contained VASt-domains. Finally Group V contained mammalian
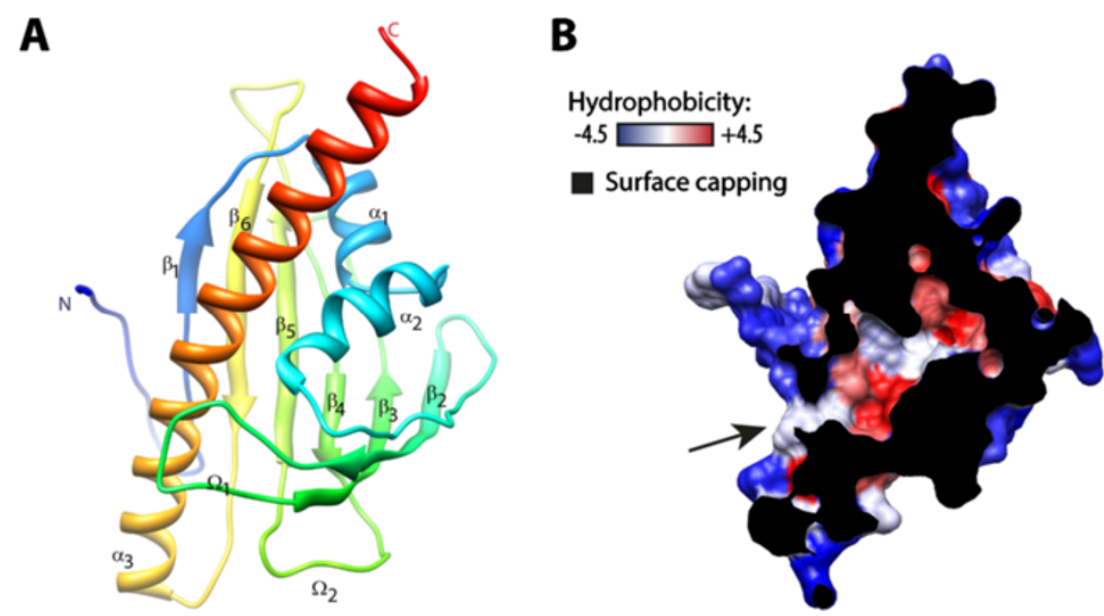

Figure 4 Homology modeling of the VASt domain 3D structure. (A) Ribbon diagram representation of the predicted 3D structure of AtVAD1 VASt domain, colored from blue ( $\mathrm{N}$-terminus) to red (C-terminus). Secondary structure elements are numbered from $\mathrm{N}$ to $\mathrm{C}$ terminus. (B) The VASt domain harbors a large hydrophobic cavity. Surface of AtVAD1 VASt model colored according to residue hydrophobicity. Capping surface is shown in black, the entrance of the hydrophobic cavity is marked by an arrow. 


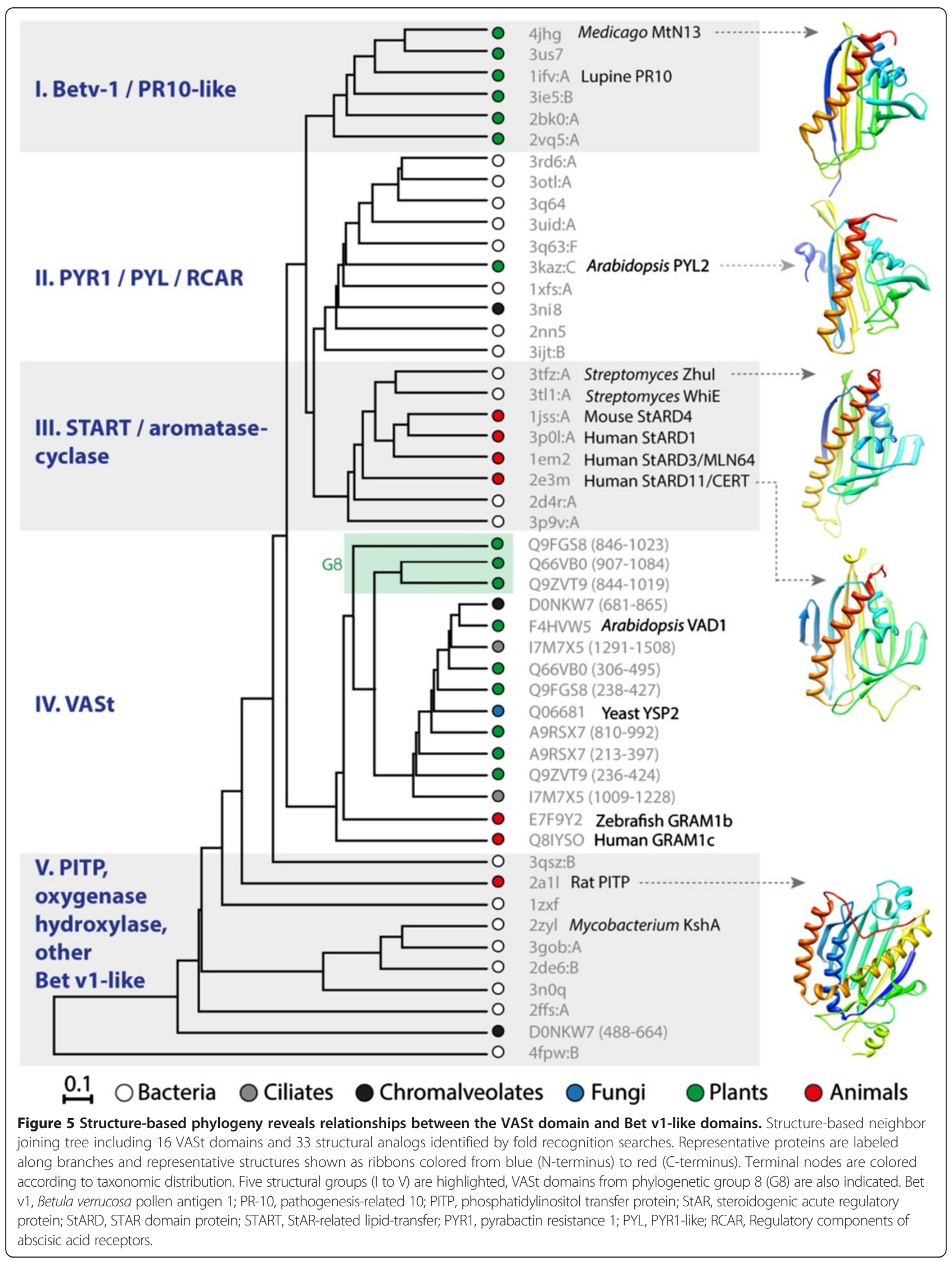


phosphatidylinositol transfer protein (PITP), bacterial oxygenase and hydrolases, and other Bet v1-like domains. With the exception of the N-terminal VASt domain of Phytophthora infestans predicted protein PITG_12663 [Uniprot:D0NKW7], all VASt domains clustered together into structural group IV. A separate sub-group within structural group IV was formed by VASt domains from sequence-based phylogenetic group 8, supporting a possible functional divergence. This analysis revealed that VASt domains are structurally related to Bet v1-like domains [Pfam:CL0209] known to bind bulky hydrophobic ligands such as phytohormones, lipids and polyketides. Association with lipid-binding domains in large multidomain proteins is typical for START and VASt domains.

To further test whether AtVAD1 VASt domain and the well-characterized START domain of the CERT protein could be evolutionary related, we closely examined the ligand-binding pockets of these two proteins. For this, we performed a structural alignment of AtVAD1 VASt and CERT START domain models. The structural alignment of AtVAD1 VASt domain and CERT START domain highlighted a good conservation of secondary structure elements lining the substrate binding pocket (Figure 6). Although the overall sequence conservation between the two proteins is limited, residues binding to C18 ceramide in CERT showed conservation or similar environments in AtVAD1 VASt domain. These observations are consistent with hidden homology between VASt and Bet v1-like domains. Alternatively, the analogy between VASt and Bet v1-like domains could result from convergent evolution. Testing the importance of the predicted ligand-binding pocket residues for VASt function could help discriminate between these hypotheses.

\section{Discussion}

We report the identification of the VASt protein domain in the VAD1 plant cell death regulator. This domain is conserved across eukaryotes and is structurally related to Bet v1-like domains, including START lipid-binding domains. The predicted structure of VAD1 VASt domain is consistent with a function in binding large hydrophobic ligands. Our findings open new perspectives for the analysis of functions of the VASt domain associated with the GRAM, C2 and PH lipid-binding domains and the characterization of novel mechanisms regulating PCD in plants.

What is the physiological role of VASt-containing proteins? Most of the proteins containing VASt domain have no characterized function to date. The $A$. thaliana VAD1 is the only exception in plants. In the context of pathogen attack, controlled programmed cell death (PCD) is one of the prevailing plant defense responses, allowing confinement of the pathogen locally in dead cells. The vad1 mutant exhibits spontaneous PCD lesions initiated in cells surrounding vascular tissue progressively expand to the whole leaf, hence its classification as "propagation lesion mimic mutant" [7]. This phenotype suggests that vad1 is impaired in the control of cell-to-cell propagation of PCD, involving a yet unknown mechanism.Amiodarone is a $\mathrm{Ca}^{2+}$ channel-targeted drug inducing apoptosis mediated by reactive oxygen species (ROS), via the same pathway as natural pheromones [27]. Genetic screens have revealed the function of the YSP2 (Yeast Suicide Protein 2) in enhancing survival after amiodarone treatment. YSP2 is a mitochondrial membrane protein involved in mitochondrial fragmentation, probably acting downstream of ROS production triggered by intracellular acidification [22]. YSP2 harbors a GRAM and a VASt domain but its molecular function is unknown. In human, a polymorphism in the GRAMD1B gene has been associated with susceptibility to chronic lymphocytic leukemia [28]. Recently, a whole genome modified-siRNA screen identified GRAMD1B as a protein associated with chemoresistance in epithelial ovarian cancer $(\mathrm{OvCa})$ cells. Consistent with

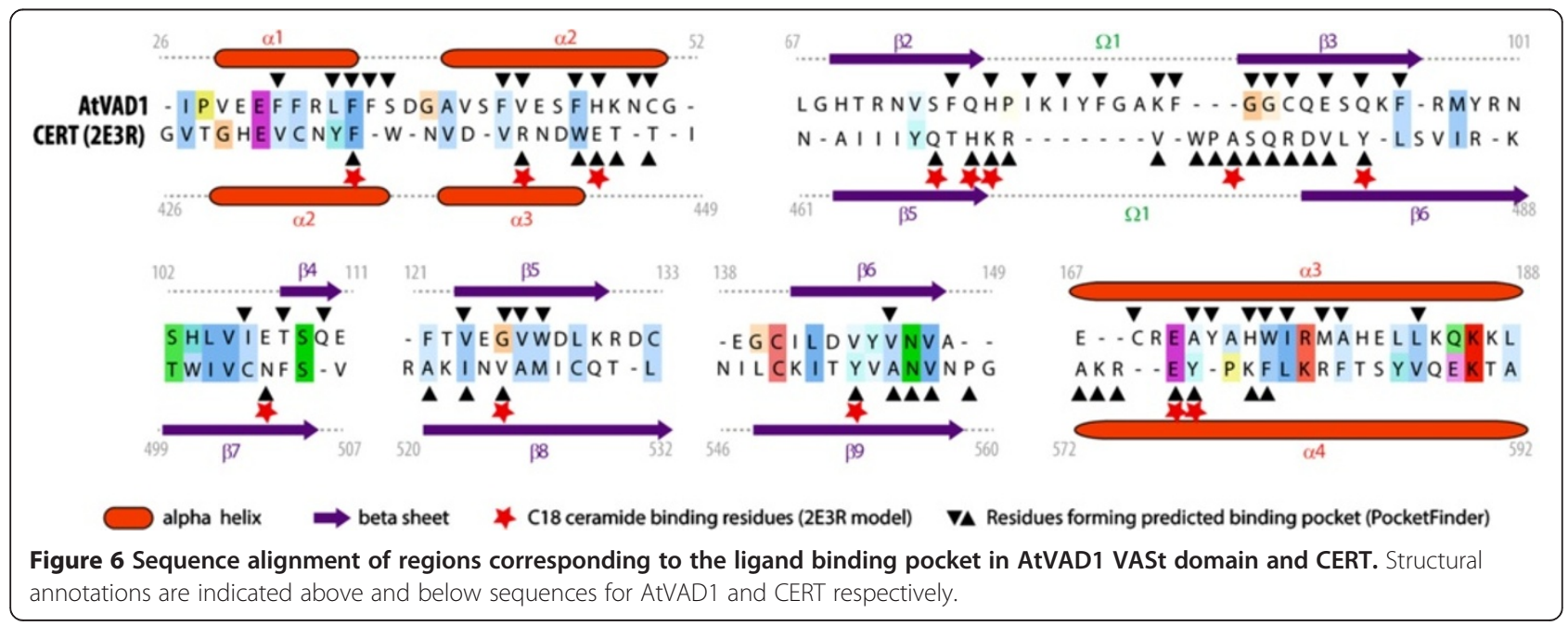


the view that acquired chemoresistance is a major contributor to patient mortality from $\mathrm{OvCa}$, reducing GRAMD1B expression increased overall survival in OvCa patients and decreased tumour burden in mouse models [29]. GRAMD1C has been identified as part of a quantitative trait locus associated with hepatic iron overload in mice, but its function has not been validated [30]. The molecular function of GRAMD1 proteins has not been investigated but their association with several disorders supports the relevance of VAStcontaining proteins for cell integrity. What may be the signal(s) associated to VAD1 that mediate propagation of PCD? The VASt domain is related to domains from the Bet v1-like superfamily [Pfam:CL0209] that bind large hydrophobic ligands such as lipids, hormones and antibiotics [31]. In the Bet v1-like superfamily, PR-10, Bet $\mathrm{v} 1$ and PYR/PYL/RCAR domains (Figure 5, groups I and II) typically bind phytohormones such as brassinosteroids, cytokinins and abscisic acid [32-34]. Some other Bet v1-like domains (Figure 5, groups III and V) bind secondary metabolites such as flavonoids, polyketides and various antibiotics $[31,35]$. These ligands are diffusible molecules that could act as intercellular signals regulated by VAD1. Domains belonging to the START subfamily of Bet v1-like domains bind lipids such as sterols and sphingolipids [31,36]. In animal cells, intercellular transport of sterols and sphingolipids is mainly mediated by non-vesicular transport via the action of dedicated lipid transport proteins (LTPs) or via spontaneous lipid exchange [37]. Phytohormone- and secondary metabolite-binding proteins in the Bet v1-like superfamily often function as single-domain proteins, or multimers of single domain-proteins, whereas START and VASt-containing proteins are generally large multidomain proteins. Notably, the mammalian CERT and Arabidopsis VAD1 proteins share a common domain structure involving a $\mathrm{PH}$ superfamily domain (PH and GRAM respectively) and a Bet v1-like superfamily domain (START and VASt respectively). Cooperation between the $\mathrm{PH}$ and START domains in CERT is critical for its function as a ceramide transport protein [38]. Ceramides and other sphingolipids are important regulators of cell death programs in animals and plants $[39,40]$. VAD1 may therefore sense or transport lipids to modulate cell death signals intercellularly.

What is the evolutionary history of the VASt domain? Our sequence- and structure-based phylogenetic analyses suggest that the VASt domain evolved from a primordial Bet v1-related protein that existed in the last universal common ancestor, and emerged with the divergence of Eukaryotes. Alternatively, the analogous structure of VASt and Bet v1-like domains could result from convergent evolution. By contrast to the PR10-like subfamily of Bet v1 domains, the VASt domain is conserved across all major Eukaryotic lineages, and therefore probably serves a function relatively conserved across all Eukaryotes. Radauer et al. proposed that the primordial Bet v1 protein would bind lipids, and would have evolved by addition of secondary structural elements or fusion to other domains into multi-domain proteins [31]. Our results suggest that the VASt domain has been associated with the GRAM domain very early in the history of Eukaryotes, and was later combined with $\mathrm{C} 2$ domains in Plants and with Pex24p domains in Oomycetes. The 3D model we obtained for VAD1 VASt domain features a long loop connecting helix $\alpha 2$ and sheet $\beta 2$, instead of a beta-sheet in typical Bet v1 domains, leading to a $\beta-\alpha 2-\beta 5-\alpha$ instead of $\beta-\alpha 2-\beta 6-\alpha$ secondary structure arrangement. VASt domains of Plants span across four phylogenetic groups (Group 3, 5, 8 and 9, Figure 3), suggesting the emergence of novel adaptations in the Plant kingdom, that may either reflect the evolution of new catalytic activities or the adaptation to plant-specific ligand(s).

\section{Conclusions}

Local variations in membrane protein and lipid composition create subcellular compartments with diverse physico-chemical properties. Such local variations in lipid and protein content may be critical for defining the specific structure membrane compartments and flagging them for the addressing of proteins and other signals [41-43]. The trafficking routes between membrane compartments, and the proteins implicated, are just starting to be uncovered. Our analyses revealed the VASt domain as a member of the Bet v1-like superfamily predominantly associated with lipid binding domains such as GRAM, C2 and $\mathrm{PH}$ domains. This finding opens new perspectives for molecular and genetics studies of the function and regulation of VASt domain containing proteins.

\section{Methods}

\section{Identification of VAD1 homologs and conservation} analysis in plants

The VAD1 protein sequence [Uniprot: F4HVW5] was used as a query for a profile Hidden Markov Model (HMM) search with phmmer [44] against the Uniprot database using Blosum62 matrix. Hits with e-value $1 \mathrm{e}^{-100}$ or less were selected and manually curated resulting in the identification of 13 homologs, exclusively from plants. Sequences were aligned using MAFFT version 7 [21] with default parameters. Amino acids conservation score, calculated according to [45], was plotted as a moving average using a sliding window approach with window size 10 and steps of size 1.Protein sequence features retrieved through the phmmer search were manually mapped along the alignment. AtVAD1 gene model (At1g02120.1) was retrieved from TAIR (www.arabidopsis.org) and mapped on the protein alignment using GeneWise (http://www.ebi.ac. uk/Tools/psa/genewise/) with default parameters. 


\section{Conservation and phylogenetic analyses of VAD1 VASt domain}

The precise boundaries of the newly identified VASt domain were set based on a conservation score $>4$ among plant AtVAD1 homologs and to include well-conserved N-terminal residues F1, D7 and P11, delimiting a 193 amino acids domain. The ungapped alignment of the 193 amino acids domain of VAD1 VASt domain with its 13 homologs was used as entry for HMM searches with jackhmmer [44] using Blosum90 matrix against NR database, with cut off e-value of $1 \mathrm{e}^{-10}$. The final list of proteins containing VASt domains was obtained after 3 iterations of jackhammer search using all hits from previous iteration as a seed. Sequence logo of the 452 VASt domains alignment was done with LOGOMAT-M [46]. To built the VASt domains phylogenetic tree, we selected all hits from 17 fully sequenced species representative of all major Eukaryotic lineages as follows: Homo sapiens and Mus musculus (Mammals), Caenorhabditis elegans and Drosophila melanogaster (Other Metazoans), Arabidopsis thaliana and Oryza sativa (Tracheophyta), Physcomitrella patens (Briophyta), Saccharomyces cerevisiae, Aspergillus nidulans, Neurospora crassa and Ustilago maydis (Fungi), Phytophthora infestans and Thalassiosira pseudonana (Stramenopiles), Entamoeba histolytica and Dictyostelium discoideum (Amoebozoa), Leishmania major (Kinetoplasts), Tetrahymena thermophila (Ciliates). After removing incomplete sequences and redundant sequences with CD-HIT [47], 85 sequences were aligned using MAFFT version 7 [21]. The alignment was automatically curated using TrimAI [48] to keep 125 positions out of 341 . Selection of LG + I + G + F as best-fit models with alpha value 2.108 for omega distribution was performed in ProtTest2 [49]. A phylogenetic tree showing branch support values as aLRT SH-like test was generated using PhyML 3.0 [50] and visualized using iTOL [51].

\section{AtVAD1 VASt domain 3D structure modeling and structure-based clustering}

The 3D structure of AtVAD1 (At1g02120) VASt domain (residues 257 to 449) was predicted with I-TASSER web server (http://zhanglab.ccmb.med.umich.edu/I-TASSER/). For structural tree inference, we first searched for AtVAD1 VASt domain structural analogs using the Vector Alignment Search Tool server [52]. Forty six analogs aligned over 100 amino-acids or more were retrieved. Second, we predicted the three dimensional of 15 VASt domains with Modeller v9.12 [53] using AtVAD1 VASt domain as a template. A structural alignment of AtVAD1 VASt domain, 15 other VASt domain structures and 46 structural analogs was generated with Mustang-MR [54]. Pairwise root-mean-square deviation (RMSD) between $\mathrm{C} \alpha$ atoms were normalized as 100*RMSD/number of aligned residues, and used to build the distance matrix for tree inference. The tree was constructed using Fitch-Margoliash method in Phylip [55] with power 2.0, and a number of terminal branches connecting uncharacterized proteins were manually removed for clarity, resulting in a distance tree with 49 structures. All protein structures were rendered with UCSF Chimera [56].

\section{Availability of supporting data}

The original version of the tree shown in Figure 3A can be accessed at http://itol.embl.de/shared/lipm_bioinfo under the project 'VAST'. All other supporting data associated with this manuscript are included as additional files.

\section{Additional files}

Additional file 1: Figure S1. Multiple sequence alignment of VASt domains from AtVAD1 and its 12 closets plant homologs. Positions showing $>70 \%$ identity are highlighted in blue.

Additional file 2: Multiple alignment file in .FASTA format of AtVAD1 and its 12 closest homologs.

Additional file 3: Table of the $\mathbf{4 5 2}$ proteins retrieved from Uniprot after HMM search using the model built from 14 VAD1 homologs as bait.

Additional file 4: Multiple alignment file in .FASTA format of 85 VAST domains.

Additional file 5: Multiple alignment file in .FASTA format of 85 VAST domains after clean-up by trimAl, used to generate the tree shown in Figure 3A.

Additional file 6: Phylogenetic tree of 85 VASt-containing proteins from 17 fully-sequenced genomes in newick format.

Additional file 7: Atomic coordinates of AtVAD1 VASt domain best model obtained via modeling by I-TASSER in .pdb format.

Additional file 8: List of AtVAD1 VASt domain closest structural analogs identified in the medium redundancy subset of the Molecular Modeling database using NCBI Vector Alignment Search Tool. Only structures that aligned over 100 residues or more were included in further analyses.

Additional file 9: Pairwise RMSD matrix used for the generation of the structure-based neighbor-joining tree of VASt domain analogs in phylip format.

Additional file 10: Structure based neighbor-joining tree of VASt domain analogs in newick format.

Competing interests

The authors declare no conflict of interests.

\section{Authors' contributions}

Conceived and designed the analyses: MK, LC, SR. Performed and interpreted the analyses: MK, LC, CB, SR. Wrote the paper: MK, CB, SR. All authors read and approved the manuscript.

\section{Acknowledgments}

We thank Penny Coggill for comments on the manuscript and referencing of our work in the Pfam database. This work has been performed in the LIPM, part of the "Laboratoire d'Excellence" (LABEX) entitled TULIP (ANR-10-LABX-41). SR is supported by a Marie Curie CIG grant (contract 334036) and a Starting Grant from the European Research Council (ERC, contract 336808).

Received: 17 January 2014 Accepted: 19 June 2014

Published: 26 June 2014 


\section{References}

1. Forslund K, Sonnhammer EL: Predicting protein function from domain content. Bioinformatics 2008, 24(15):1681-1687.

2. Söding J, Lupas AN: More than the sum of their parts: on the evolution of proteins from peptides. Bioessays 2003, 25(9):837-846.

3. Chothia C, Gough J, Vogel C, Teichmann SA: Evolution of the protein repertoire. Science 2003, 300(5626):1701-1703.

4. Vogel C, Berzuini C, Bashton M, Gough J, Teichmann SA: Supra-domains: evolutionary units larger than single protein domains. J Mol Biol 2004, 336(3):809-823.

5. Lorrain S, Vailleau F, Balagué C, Roby D: Lesion mimic mutants: keys for deciphering cell death and defense pathways in plants? Trends Plant Sci 2003, 8(6):263-271.

6. Moeder W, Yoshioka K: Lesion mimic mutants: a classical, yet still fundamental approach to study programmed cell death. Plant Signal Behav 2008, 3(10):764-767.

7. Lorrain S, Lin B, Auriac MC, Kroj T, Saindrenan P, Nicole M, Balagué C, Roby D: vascular associated death1, a novel GRAM domain-containing protein, is a regulator of cell death and defense responses in vascular tissues. Plant Cell 2004, 16(8):2217-2232.

8. Bouchez O, Huard C, Lorrain S, Roby D, Balague C: Ethylene is one of the key elements for cell death and defense response control in the Arabidopsis lesion mimic mutant vad1. Plant Physiol 2007, 145(2):465-477.

9. Doerks T, Strauss M, Brendel M, Bork P: GRAM, a novel domain in glucosyltransferases, myotubularins and other putative membrane-associated proteins. Trends Biochem Sci 2000, 25(10):483-485.

10. Berger P, Schaffitzel C, Berger I, Ban N, Suter U: Membrane association of myotubularin-related protein 2 is mediated by a pleckstrin homology-GRAM domain and a coiled-coil dimerization module. Proc Natl Acad Sci USA 2003, 100(21):12177-12182.

11. Tsujita K, Itoh T, ljuin T, Yamamoto A, Shisheva A, Laporte J, Takenawa T: Myotubularin regulates the function of the late endosome through the gram domain-phosphatidylinositol 3, 5-bisphosphate interaction. J Biol Chem 2004, 279(14):13817-13824.

12. S-i Y, Oku M, Wasada Y, Ano Y, Sakai Y: PI4P-signaling pathway for the synthesis of a nascent membrane structure in selective autophagy. J Cell Biol 2006, 173(5):709-717.

13. Jiang $S-Y$, Ramamoorthy R, Ramachandran S: Comparative transcriptional profiling and evolutionary analysis of the GRAM domain family in eukaryotes. Dev Biol 2008, 314(2):418-432.

14. Kachroo P, Shanklin J, Shah J, Whittle EJ, Klessig DF: A fatty acid desaturase modulates the activation of defense signaling pathways in plants. Proc Natl Acad Sci USA 2001, 98(16):9448-9453.

15. Brodersen P, Petersen M, Pike HM, Olszak B, Skov S, Ødum N, Jørgensen LB, Brown RE, Mundy J: Knockout of Arabidopsis accelerated-cell-death11 encoding a sphingosine transfer protein causes activation of programmed cell death and defense. Genes Dev 2002, 16(4):490-502.

16. Liang H, Yao N, Song JT, Luo S, Lu H, Greenberg JT: Ceramides modulate programmed cell death in plants. Genes Dev 2003, 17(21):2636-2641.

17. Tang D, Ade J, Frye CA, Innes RW: Regulation of plant defense responses in Arabidopsis by EDR2, a PH and START domain-containing protein. Plant J 2005, 44(2):245-257.

18. Wang W, Yang X, Tangchaiburana S, Ndeh R, Markham JE, Tsegaye Y, Dunn TM, Wang G-L, Bellizzi M, Parsons JF: An inositolphosphorylceramide synthase is involved in regulation of plant programmed cell death associated with defense in Arabidopsis. Plant Cell 2008, 20(11):3163-3179.

19. Vorwerk S, Schiff C, Santamaria M, Koh S, Nishimura M, Vogel J, Somerville C, Somerville S: EDR2 negatively regulates salicylic acid-based defenses and cell death during powdery mildew infections of Arabidopsis thaliana. BMC Plant Biol 2007, 7(1):35.

20. Punta M, Coggill PC, Eberhardt RY, Mistry J, Tate J, Boursnell C, Pang N, Forslund K, Ceric G, Clements J, Heger A, Holm L, Sonnhammer EL, Eddy SR, Bateman A, Finn RD: The Pfam protein families database. Nucleic Acids Res 2012, 40(D1):D290-D301.

21. Katoh K, Misawa K, Kuma K-i, Miyata T: MAFFT: a novel method for rapid multiple sequence alignment based on fast fourier transform. Nucleic Acids Res 2002, 30(14):3059-3066.

22. Sokolov S, Knorre D, Smirnova E, Markova O, Pozniakovsky A, Skulachev V, Severin F: Ysp2 mediates death of yeast induced by amiodarone or intracellular acidification. Biochim Biophys Acta Bioenerg 2006, 1757(9):1366-1370.
23. Lundin D, Poole AM, Sjöberg B-M, Högbom M: Use of structural phylogenetic networks for classification of the ferritin-like superfamily. J Biol Chem 2012, 287(24):20565-20575.

24. Zhang Y: I-TASSER server for protein 3D structure prediction. BMC Bioinformatics 2008, 9(1):40.

25. Iyer LM, Koonin EV, Aravind L: Adaptations of the helix-grip fold for ligand binding and catalysis in the START domain superfamily. Protein Struct Funct Genet 2001, 43(2):134-144.

26. Roy A, Kucukural A, Zhang Y: I-TASSER: a unified platform for automated protein structure and function prediction. Nat Protoc 2010, 5(4):725-738.

27. Pozniakovsky Al, Knorre DA, Markova OV, Hyman AA, Skulachev VP, Severin FF: Role of mitochondria in the pheromone-and amiodarone-induced programmed death of yeast. J Cell Biol 2005, 168(2):257-269.

28. Di Bernardo MC, Crowther-Swanepoel D, Broderick P, Webb E, Sellick G, Wild R, Sullivan K, Vijayakrishnan J, Wang Y, Pittman AM, Sunter NJ, Hall AG, Dyer MJS, Matutes E, Dearden C, Mainou-Fowler T, Jackson GH, Summerfield G, Harris RJ, Pettitt AR, Hillmen P, Allsup DJ, Bailey JR, Pratt G, Pepper C, Fegan C, Allan JM, Catovsky D, Houlston RS: A genome-wide association study identifies six susceptibility loci for chronic lymphocytic leukemia. Nat Genet 2008, 40(10):1204-1210.

29. Wu SY, Yang X, Gharpure KM, Hatakeyama H, Egli M, McGuire MH, Nagaraja AS, Miyake TM, Rupaimoole R, Pecot CV, Taylor M, Pradeep S, Sierant M, Rodriguez-Aguayo C, Choi HJ, Previs RA, Armaiz-Pena GN, Huang L, Martinez C, Hassell T, Ivan C, Sehgal V, Singhania R, Han HD, Su C, Kim JH, Dalton HJ, Kovvali C, Keyomarsi K, McMillan NAJ, Overwijk WW, Liu J, Lee JS, Baggerly KA, Lopez-Berestein G, Ram PT, Nawrot B, Sood AK: 2'-OMe-phosphorodithioate-modified siRNAs show increased loading into the RISC complex and enhanced anti-tumour activity. Nat Commun 2014, 5:1-12.

30. Guo X, Zhang Z, Zhang F, Tao Y, An P, Wu Q, Wang C-Y, Knutson MD, Wang F: Fine-mapping and genetic analysis of the loci affecting hepatic iron overload in mice. PLoS One 2013, 8(5):e63280.

31. Radauer C, Lackner P, Breiteneder H: The Bet $v \mathbf{1}$ fold: an ancient, versatile scaffold for binding of large, hydrophobic ligands. BMC Evol Biol 2008, 8(1):286

32. Marković-Housley Z, Degano M, Lamba D, von Roepenack-Lahaye E, Clemens S, Susani M, Ferreira F, Scheiner O, Breiteneder H: Crystal structure of a hypoallergenic isoform of the major birch pollen allergen Bet $\mathrm{v} 1$ and its likely biological function as a plant steroid carrier. J Mol Biol 2003, 325(1):123-133.

33. Fernandes $\mathrm{H}$, Michalska K, Sikorski M, Jaskolski M: Structural and functional aspects of PR-10 proteins. FEBS J 2013, 280(5):1169-1199.

34. Miyakawa T, Fujita Y, Yamaguchi-Shinozaki K, Tanokura M: Structure and function of abscisic acid receptors. Trends Plant Sci 2012.

35. Lee E-J, Facchini P: Norcoclaurine synthase is a member of the pathogenesis-related 10/Bet v1 protein family. Plant Cell 2010, 22(10):3489-3503.

36. Alpy F, Tomasetto C: Give lipids a START: the StAR-related lipid transfer (START) domain in mammals. J Cell Sci 2005, 118(13):2791-2801.

37. Lev S: Non-vesicular lipid transport by lipid-transfer proteins and beyond. Nat Rev Mol Cell Biol 2010, 11(10):739-750.

38. Kumagai K, Kawano M, Shinkai-Ouchi F, Nishijima M, Hanada K: Interorganelle trafficking of ceramide is regulated by phosphorylation-dependent cooperativity between the PH and START domains of CERT. J Biol Chem 2007, 282(24):17758.

39. Young MM, Kester M, Wang H-G: Sphingolipids: regulators of crosstalk between apoptosis and autophagy. J Lipid Res 2013, 54(1):5-19.

40. Berkey R, Bendigeri D, Xiao S: Sphingolipids and plant defense/disease: the "death" connection and beyond. Front Plant Sci 2012, 3:68.

41. Tilsner J, Amari K, Torrance L: Plasmodesmata viewed as specialised membrane adhesion sites. Protoplasma 2011, 248(1):39-60.

42. Ehlers K, Westerloh MG: Developmental Control of Plasmodesmata Frequency, Structure, and Function. In Symplasmic Transport in Vascular Plants. Science+Business Media New York: Springer; 2013:41-82.

43. Perraki A, Cacas JL, Crowet JM, Lins L, Castroviejo M, German-Retana S, Mongrand S, Raffaele S: Plasma Membrane Localization of Solanum tuberosum Remorin from Group 1, Homolog 3 is Mediated by Conformational Changes in a Novel C-Terminal Anchor and Required for the Restriction of Potato Virus X Movement. Plant Physiol 2012, 160(2):624-637.

44. Finn RD, Clements J, Eddy SR: HMMER web server: interactive sequence similarity searching. Nucleic Acids Res 2011, 39(suppl 2):W29-W37. 
45. Livingstone $C D$, Barton GJ: Protein sequence alignments: a strategy for the hierarchical analysis of residue conservation. Comput Appl Biosci 1993, 9(6):745-756.

46. Schuster-Böckler B, Schultz J, Rahmann S: HMM Logos for visualization of protein families. Bioinformatics 2004, 5(1):7.

47. Li W, Godzik A: Cd-hit: a fast program for clustering and comparing large sets of protein or nucleotide sequences. Bioinformatics 2006, 22(13):1658-1659.

48. Capella-Gutiérrez S, Silla-Martínez JM, Gabaldón T: TrimAl: a tool for automated alignment trimming in large-scale phylogenetic analyses. Bioinformatics 2009, 25(15):1972-1973.

49. Abascal F, Zardoya R, Posada D: ProtTest: selection of best-fit models of protein evolution. Bioinformatics 2005, 21(9):2104-2105.

50. Guindon S, Dufayard J-F, Lefort V, Anisimova M, Hordijk W, Gascuel O: New algorithms and methods to estimate maximum-likelihood phylogenies: assessing the performance of PhyML 3.0. Syst Biol 2010, 59(3):307-321.

51. Letunic I, Bork P: Interactive tree of life v2: online annotation and display of phylogenetic trees made easy. Nucleic Acids Res 2011, 39(suppl 2):W475-W478.

52. Gibrat J-F, Madej T, Bryant SH: Surprising similarities in structure comparison. Curr Opin Struct Biol 1996, 6(3):377-385.

53. Sali A, Blundell TL: Comparative protein modelling by satisfaction of spatial restraints. J. Mol. Biol. 1993, 234:779-815.

54. Konagurthu AS, Reboul CF, Schmidberger JW, Irving JA, Lesk AM, Stuckey PJ, Whisstock JC, Buckle AM: MUSTANG-MR structural sieving server: applications in protein structural analysis and crystallography. PLoS One 2010, 5(4):e10048.

55. Felsenstein J: PHYLIP - phylogeny inference package (version 3.2). Cladistics 1989, 5:164-166.

56. Pettersen EF, Goddard TD, Huang CC, Couch GS, Greenblatt DM, Meng EC, Ferrin TE: UCSF chimera-a visualization system for exploratory research and analysis. J Comput Chem 2004, 25(13):1605-1612.

doi:10.1186/1471-2105-15-222

Cite this article as: Khafif et al:: Identification and phylogenetic analyses of VASt, an uncharacterized protein domain associated with lipid-binding domains in Eukaryotes. BMC Bioinformatics 2014 15:222.

\section{Submit your next manuscript to BioMed Central and take full advantage of:}

- Convenient online submission

- Thorough peer review

- No space constraints or color figure charges

- Immediate publication on acceptance

- Inclusion in PubMed, CAS, Scopus and Google Scholar

- Research which is freely available for redistribution 